\title{
Riccati PDEs That Imply Curvature-Flatness
}

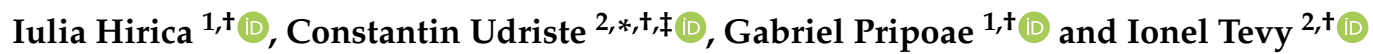 \\ 1 Faculty of Mathematics and Computer Science, University of Bucharest, Academiei 14, Sector 1, \\ RO-010014 Bucharest, Romania; ihirica@fmi.unibuc.ro (I.H.); gpripoae@yahoo.com (G.P.) \\ 2 Department of Mathematics and Informatics, Faculty of Applied Sciences, University Politehnica \\ of Bucharest, Splaiul Independentei 313, Sector 6, RO-060042 Bucharest, Romania; tevy@mathem.pub.ro \\ * Correspondence: udriste@mathem.pub.ro; Tel.: +40-745-371-684 \\ + These authors contributed equally to this work. \\ $\ddagger$ Second address: Academy of Romanian Scientists, Ilfov 3, Sector 5, RO-050044 Bucharest, Romania.
}

Citation: Hirica, I.; Udriste, C.;

Pripoae, G.; Tevy, I. Riccati PDEs That Imply Curvature-Flatness.

Mathematics 2021, 9, 537. https://

doi.org/10.3390/math9050537

Academic Editor: George

Kaimakamis

Received: 5 February 2021

Accepted: 26 February 2021

Published: 4 March 2021

Publisher's Note: MDPI stays neutral with regard to jurisdictional claims in published maps and institutional affiliations.

Copyright: (c) 2021 by the authors. Licensee MDPI, Basel, Switzerland. This article is an open access article distributed under the terms and conditions of the Creative Commons Attribution (CC BY) license (https:// creativecommons.org/licenses/by/ $4.0 /)$.

\begin{abstract}
In this paper the following three goals are addressed. The first goal is to study some strong partial differential equations (PDEs) that imply curvature-flatness, in the cases of both symmetric and non-symmetric connection. Although the curvature-flatness idea is classic for symmetric connection, our main theorems about flatness solutions are completely new, leaving for a while the point of view of differential geometry and entering that of PDEs. The second goal is to introduce and study some strong partial differential relations associated to curvature-flatness. The third goal is to introduce and analyze some vector spaces of exotic objects that change the meaning of a generalized Kronecker delta projection operator, in order to discover new PDEs implying curvature-flatness. Significant examples clarify some ideas.
\end{abstract}

Keywords: curvature-flatness PDEs; Riccati PDEs; generalized Kronecker delta; eigentensors; metrics and connections adapted to flatness

MSC: 58J99; 53C44; 53C21

\section{Introduction and Contributions}

Differential geometry is often considered "the art of manipulating partial differential equations (PDEs)". This point of view can be found in the papers [1-12], which develop the following topics: general theory of PDEs, symmetries and overdetermined systems of PDEs, fully nonlinear equations on Riemannian manifolds with negative curvature, basic evolution PDEs in Riemannian geometry, foundations of differential geometry, affine differential geometry, overdetermined systems of linear PDEs, geometric dynamics on Riemannian manifolds, the role of PDEs in differential geometry, the Dirichlet problem for first-order PDEs, and differential inclusions.

Specific aims in this paper are as follows: (i) introducing and analyzing those PDEs or partial differential relations to define and study curvature-flatness; (ii) developing techniques to find intrinsic properties of some diagnostic PDEs; (iii) discussing explicit examples to illustrate the importance of the choice of an appropriate research technique.

To underline our original point of view, we try to impose the following direction of research: instead of looking for a coordinate system in which the connection $\Gamma_{j k}^{i}$ is a solution of the tensor PDEs $R_{i j k}^{l}=0$, we are looking just for connections that are solutions of these PDEs, and we then analyze whether the solutions are linked by variable changes.

In this paper we analyze the PDEs required to determine whether a geometric manifold (endowed either with a metric or with a linear connection) is flat or curved. The method presented is to find a diagnostic PDE system whose solutions indicate that the manifold is flat. The diagnostic that we are looking for is a Riccati PDE system. Although the PDEs of curvature-flatness are tensor equations (obviously coordinate-independent), some of the 
associated Riccati PDEs are not. The nonlinear PDEs in this paper are called Riccati-like because they arise in a similar way as Riccati ordinary differential equations (ODEs).

It is our hope that this paper, taken as a whole, will provide a broad overview of geometry and its relationship to PDEs. The subjects of the constructive Sections are as follows: (2) curvature-flatness connections (symmetric connection case, vector spaces of exotic objects, strong Riccati PDEs, non-symmetric connection case), (3) curvature-flat Riemannian manifolds (properties of a projection operator; strong Riccati PDE system; giving the curvature tensor field, finding the Riemannian metric), (4) invariant version for curvature-flatness (symmetric connection case, non-symmetric connection case), (5) conclusions. The linearization of the curvature-flatness PDE system and some examples created with the software package Maple suggest reasons for future research. The invariant technique used in Section 4 hides the curvature-flatness PDE system in differentiable identities readable only by geometers. The vector spaces of exotic objects introduced by us to give another meaning to the generalized Kronecker-type projector are a novelty worthy of use in the future.

\section{Curvature-Flatness Connections}

Curvature-flatness was discussed in the papers [13-15] (compact flat Riemannian manifolds), Ref. [16] (some regularity theorems in Riemannian geometry), Ref. [17] (local existence of smooth metrics with prescribed curvature), Ref. [18] (least squares approximation of flatness on Riemannian manifolds), and [19] (flat Riemannian manifolds), based on the idea of finding an adapted coordinate system wherein the components of the curvature tensor field and the torsion tensor field are zero. We bring up another point of view: based on some associated strong PDEs whose solutions are suitable connections and metrics, we ensure the cancellation of the curvature tensor field.

The concept of connections that determine representations of zero curvature as solutions of a given PDE system has seen renewed interest recently (see also, Ref. [20]connections defining representations of zero curvature and their Lax and Bäcklund mappings, Ref. [21] —normal forms of two-dimensional metrics admitting two projective vector fields, Ref. [22]—conformally flat submanifolds in spheres and integrable systems).

\subsection{Symmetric Connection Case}

Let us consider the pair $(M, \nabla)$, where $M$ is a smooth real $n$-dimensional manifold and $\nabla$ is an affine symmetric connection, with the components $\Gamma_{j k}^{i}, i, j, k=\overline{1, n}$, in $\mathcal{F}(M)$ (see also, [6,7]).

The connection $\nabla$ determines the curvature tensor field Riem $\nabla$ whose components are (in Einstein notation of summation)

$$
\begin{gathered}
R_{i j k}^{l}=\frac{\partial}{\partial x^{j}} \Gamma_{i k}^{l}-\frac{\partial}{\partial x^{k}} \Gamma_{i j}^{l}+\Gamma_{j m}^{l} \Gamma_{i k}^{m}-\Gamma_{k m}^{l} \Gamma_{i j}^{m} \\
=\left(\delta_{j}^{p} \delta_{k}^{s}-\delta_{k}^{p} \delta_{j}^{s}\right)\left(\frac{\partial}{\partial x^{p}} \Gamma_{i s}^{l}+\Gamma_{p m}^{l} \Gamma_{i s}^{m}\right) \\
=\left(\delta_{j}^{p} \delta_{k}^{s}-\delta_{k}^{p} \delta_{j}^{s}\right)\left(\frac{\partial}{\partial x^{p}} \Gamma_{i s}^{l}-\Gamma_{s m}^{l} \Gamma_{i p}^{m}\right),
\end{gathered}
$$

where $i, j, k, l=\overline{1, n}$. The basic properties of curvature tensor field include

$$
\begin{gathered}
R_{i j k}^{l}+R_{i k j}^{l}=0, R_{i j k}^{l}+R_{j k i}^{l}+R_{k i j}^{l}=0 \\
R_{i j k, m}^{l}+R_{i k m, j}^{l}+R_{i m j, k}^{l}=0 \text { (Bianchi); } R_{i j k, m}^{l}+R_{k i m, j}^{l}+R_{m k j, i}^{l}+R_{j m i, k}^{l}=0 \text { (Veblen). }
\end{gathered}
$$

The generalized Kronecker delta $P_{j k}^{p s}=\frac{1}{2}\left(\delta_{j}^{p} \delta_{k}^{s}-\delta_{k}^{p} \delta_{j}^{s}\right)$ is a projection $P$, i.e., $P^{2}=P$, and is covariant constant (see also [23]). The natural domain of definition of the operator $P$ is the vector space of the tensor fields $\mathcal{T}_{q}^{p}(M)$, where $(p, q)=$ fixed, $p \geq 2$, or $q \geq 2$. The set 
of eigenvalues of a projector contains the eigenvalues 0 and 1. Explicitly: (i) on $\mathcal{T}_{2}^{0}(M)$, all eigentensors, respectively eigenvalues, are $\omega_{p s}$-symmetric in $(p, s)$, with $\lambda=0 ; \omega_{p s}$ antisymmetric in $(p, s)$ with $\lambda=1$; particularly, the electromagnetic tensor field $F_{p s}=$ $\partial_{p} A_{s}-\partial_{s} A_{p}$; (ii) on $\mathcal{T}_{4}^{0}(M)$, the elasticity tensor field $E_{i j k l}\left(E_{i j k l}=E_{j i k l}, E_{i j k l}=E_{i j l k}, E_{i j k l}=\right.$ $E_{k l i j}$ ) is an eigentensor corresponding to the eigenvalue 0 ; (iii) on $\mathcal{T}_{3}^{1}(M)$, the curvature tensor field is an eigentensor corresponding to the eigenvalue 1, etc. (for an eigentensor, see also [24]). As can be seen from the previous formulas, the operator $P$ also acts on exotic objects of type $\frac{\partial}{\partial x^{p}} \Gamma_{i s}^{l}+\Gamma_{p m}^{l} \Gamma_{i s}^{m}$, a situation that will be explained further. Mathematical concepts with real intrinsic geometric significance, other than connections and tensors, are called exotic objects.

The following Definition and Theorem are well-known [13-15,19].

Definition 1. The pair $(M, \nabla)$ is called curvature-flat if $\operatorname{Riem}^{\nabla}=0$.

Theorem 1. Let us consider the curvature-flatness PDE system Riem $\nabla=0$. The symmetric connection $\nabla$ is a solution if and only if it is locally Euclidean, i.e., around each point of $M$ there exists a chart such that $\Gamma_{j k}^{i}=0$.

According to this Theorem, a curvature-flat manifold is one that "locally looks like a Euclidean space". The question of whether a geometric manifold is curvature-flat depends on its connection structure and only indirectly on its topology. A topological space equipped with one connection may be flat, but equipped with another it may not be.

Remark 1. The curvature-flat PDE system $R_{i j k}^{l}(x)=0$, with the unknown $\Gamma_{j k}^{i}(x)$, is nonlinear. The linearization around a solution $\Gamma_{j k}^{i}(x)$ can be obtained in the following way: consider a variation $\Gamma_{j k}^{i}(x, t)$ of the solution $\Gamma_{j k}^{i}(x, 0)=\Gamma_{j k}^{i}(x)$; applying the differential operator $\partial_{t}$ to PDEs $R_{i j k}^{l}(x, t)=0$, denoting $\left.\partial_{t} \Gamma_{j k}^{i}(x, t)\right|_{t=0}=\xi_{j k}^{i}(x)$ (symmetric tensor field), we find the linear PDE system

$$
\partial_{j} \xi_{i k}^{l}-\partial_{k} \xi_{i j}^{l}+\xi_{j m}^{l} \Gamma_{i k}^{m}+\Gamma_{j m}^{l} \xi_{i k}^{m}-\xi_{k m}^{l} \Gamma_{i j}^{m}-\Gamma_{k m}^{l} \xi_{i j}^{m}=0,
$$

whose unknown is the symmetric tensor field $\xi_{j k}^{i}(x)$.

The motivation of linearization of a nonlinear PDE is based on the fact that in modern problems we are obliged to organize the set of solutions of a PDE as an infinite dimensional differentiable manifold, and at one point to find the tangent manifold.

Due to the fact that the curvature tensor field of components $R_{j k l}^{i}$ is zero everywhere, it can be considered as a first order tensor Riccati PDE system written in the form (see [25]) $2 P^{p s} \frac{\partial}{\partial x^{p}} \Gamma_{i s}^{l}=\Gamma_{k m}^{l} \Gamma_{i j}^{m}-\Gamma_{j m}^{l} \Gamma_{i k}^{m}$ or as a Riccati PDE system of divergence type $\frac{\partial}{\partial x^{p}}\left(\delta_{j}^{p} \Gamma_{i k}^{l}-\right.$ $\left.\delta_{k}^{p} \Gamma_{i j}^{l}\right)=\Gamma_{k m}^{l} \Gamma_{i j}^{m}-\Gamma_{j m}^{l} \Gamma_{i k}^{m}$. Since the curvature tensor field is antisymmetric in the last two indices and satisfies the first Bianchi identity, each of the previous PDE systems consists of $\frac{n^{2}\left(n^{2}-1\right)}{3}$ distinct first-order non-linear quadratic PDEs whose unknowns are $\frac{n^{2}(n+1)}{2}$ functions $\Gamma_{j k}^{i}$; for $n=2$, an undetermined system; for $n \geq 3$, an overdetermined system.

Example 1. (i) Every one-dimensional $(M, \nabla)$ manifold is flat.

(ii) For $n=2$, we have a system of four PDEs

$$
R_{i 12}^{l}=\partial_{1} \Gamma_{i 2}^{l}-\partial_{2} \Gamma_{i 1}^{l}+\Gamma_{1 m}^{l} \Gamma_{i 2}^{m}-\Gamma_{2 m}^{l} \Gamma_{i 1}^{m}=0 ; l, i=1,2,
$$

with six unknown functions $\Gamma_{11}^{1}, \Gamma_{12}^{1}, \Gamma_{22}^{1}, \Gamma_{11}^{2}, \Gamma_{12}^{2}, \Gamma_{22}^{2}$. This system has an infinity of solutions (as an example, a PDE system in unknowns $\Gamma_{12}^{1}, \Gamma_{12}^{2}$, and four arbitrary functions $\Gamma_{11}^{1}, \Gamma_{22}^{1}$, $\left.\Gamma_{11}^{2}, \Gamma_{22}^{2}\right)$. 
Case 1: Ad-hoc solution Setting $\Gamma_{11}^{1}=\Gamma_{22}^{1}=\Gamma_{11}^{2}=\Gamma_{22}^{2}=0$, and denoting $\Gamma_{12}^{1}=u$, $\Gamma_{12}^{2}=v$, we obtain a (completely integrable) gradient Riccati PDE system of the form

$$
u_{x}+u v=0, u_{y}+u^{2}=0, v_{x}+v^{2}=0, v_{y}+u v=0,
$$

having the solution

$$
u(x, y)=\frac{b}{a x+b y+c}, v(x, y)=\frac{a}{a x+b y+c}
$$

where $(x, y) \in \mathbb{R}^{2}, a x+b y+c \neq 0$.

Case 2: Ad-hoc solution: $\Gamma_{12}^{1}=\Gamma_{12}^{2}=\Gamma_{22}^{1}=\Gamma_{11}^{2}=0, \Gamma_{11}^{1}=f(x), \Gamma_{22}^{2}=g(y)$.

Case 3: Constant connection solution: Let $\Gamma_{11}^{1}=a, \Gamma_{12}^{1}=b, \Gamma_{22}^{1}=c, \Gamma_{11}^{2}=e, \Gamma_{12}^{2}=f$, and $\Gamma_{22}^{2}=g$ be constant functions. We find an algebraic system $b g+a c=b^{2}+c f, a f+e g=$ $e b+f^{2}, e c=b f$, which comes from $\frac{b}{c}=\frac{f-a}{g-b}=\frac{e}{f}$. The solution is $b=\lambda c, e=\lambda f, a=$ $f-\lambda g+\lambda^{2} c, \forall \lambda, c, f, g \in \mathbb{R}$.

(iii) Generally, a constant symmetric connection of components $\Gamma_{j k}^{i}(x)=C_{j k}^{i}$ is a curvatureflat solution if $C_{k m}^{l} C_{i j}^{m}$ is symmetric in the pair $(k, j)$. In addition, a symmetric connection $\Gamma_{j k}^{i}(x)$, for which $\Gamma_{k m}^{l}(x) \Gamma_{i j}^{m}(x)$ is symmetric in the pair $(k, j)$, is a curvature-flat solution if and only if $\Gamma_{i k}^{l}(x)=\frac{\partial f_{i}^{l}}{\partial x^{k}}(x)$, with a suitable matrix of elements $f_{i}^{l}(x)$.

The tensor Riccati PDE system $R^{i}{ }_{j k l}=0$ is equivalent to each of the two first-order Riccati PDE systems: $P_{j k}^{p s}\left(\frac{\partial}{\partial x^{p}} \Gamma_{i s}^{l}+\Gamma_{p m}^{l} \Gamma_{i s}^{m}\right)=0$ or $P^{p s}\left(\frac{\partial}{\partial x^{p}} \Gamma_{i s}^{l}-\Gamma_{s m}^{l} \Gamma_{i p}^{m}\right)=0$. There is no general algorithm for finding exact solutions of the previous PDE systems but some methods used in other areas can be useful. For example, finding solutions with a specified type.

\subsection{Vector Spaces of Exotic Objects}

Let us consider again the projector $P$ of components $P_{j k}^{p s}=\frac{1}{2}\left(\delta_{j}^{p} \delta_{k}^{s}-\delta_{k}^{p} \delta_{j}^{s}\right)$. Instead of its natural domain of definition $\mathcal{T}_{q}^{p}(M)$, where $(p, q)=$ fixed, $p \geq 2$ or $q \geq 2$, we create a non-trivial vector space of exotic objects. First we define four exotic objects as $A=\left(\partial_{x} \Gamma_{i s}^{l}\right)$, $B=\left(\partial_{x^{s}} \Gamma_{i p}^{l}\right), C=\left(\Gamma_{p m}^{l} \Gamma_{i s}^{m}\right), D=\left(\Gamma_{s m}^{l} \Gamma_{i p}^{m}\right)$, with four-indexed components, where $l, i, p, s \in \overline{1, n}$. Then we build the vector space (linear covering)

$$
\mathbb{V}=\left\{a A+b B+c C+d D \mid(a, b, c, d) \in \mathbb{R}^{4}\right\},
$$

whose four-indexed elements are

$$
V_{i p s}^{l}=a \partial_{x p} \Gamma_{i s}^{l}+b \partial_{x^{s}} \Gamma_{i p}^{l}+c \Gamma_{p m}^{l} \Gamma_{i s}^{m}+d \Gamma_{s m}^{l} \Gamma_{i p}^{m}(a, b, c, d) \in \mathbb{R}^{4}
$$

The projector $P$ acts on $\mathbb{V}$, but it is more interesting acting either on $\operatorname{Sp}\{A, C\}$ or on $S p\{B, D\}$.

The curvature tensor field $R_{i p s}^{l}$, i.e., $(a, b, c, d)=(1,-1,1,-1)$, belongs to the set $\mathbb{V}$. The set of all elements having the same symmetries as a curvature tensor field is a vector subspace

$$
\mathbb{W}=\left\{W^{l}{ }_{i j k}=a \partial_{x^{j}} \Gamma_{i k}^{l}-a \partial_{x^{k}} \Gamma_{i j}^{l}+c \Gamma_{j m}^{l} \Gamma_{i k}^{m}-c \Gamma_{k m}^{l} \Gamma_{i j}^{m} \mid(a,-a, c,-c) \in \mathbb{R}^{4}\right\} .
$$

The element $W_{i j k}^{l} \in \mathbb{W}$ is just the curvature tensor field iff $a=c=1$; if $a=$ $c \neq 1$, then $W^{l}{ }_{i j k}$ is collinear to the curvature tensor field. Otherwise, $W^{l}{ }_{i j k}$ is an exotic object (non-tensor). 
The kernel and the image of the projector $P: \mathbb{V} \rightarrow \mathbb{V}$ are $\operatorname{ker}(P)=\left\{V_{i j k}^{l}\right.$, symmetric in $\left.(j k)\right\}, \operatorname{im}(P)=\left\{Y_{i j k}^{l}=\frac{1}{2}\left(V_{i j k}^{l}-V_{i k j}^{l}\right)\right.$, antisymmetric in $\left.(j k)\right\}$. Equivalently, $\operatorname{ker}(P): a=b, c=d ; \operatorname{im}(P): a=-b, c=-d$.

Proposition 1. The curvature tensor field belongs to im $(P)$.

\subsection{Strong Riccati PDEs}

For zero-curvature equations having unknown connection components it is very difficult to highlight and find non-trivial solutions. Consequently we look for strong PDEs that give indirect solutions of the curvature-flatness PDEs.

Example 2. (i) If $\frac{\partial}{\partial x j} \Gamma_{i k}^{l}+\Gamma_{j m}^{l} \Gamma_{i k}^{m}=0$, then $\frac{\partial}{\partial x^{k}} \Gamma_{i j}^{l}+\Gamma_{k m}^{l} \Gamma_{i j}^{m}=0$ and hence $R_{i j k}^{l}=0$. (ii) If $\frac{\partial}{\partial x^{j}} \Gamma_{i k}^{l}-\Gamma_{k m}^{l} \Gamma_{i j}^{m}=0$, then $-\frac{\partial}{\partial x^{k}} \Gamma_{i j}^{l}+\Gamma_{j m}^{l} \Gamma_{i k}^{m}=0$ and hence $R_{i j k}^{l}=0$.

Let us now discuss some Riccati PDEs that imply curvature-flatness.

Theorem 2. Let $\nabla$ be a symmetric connection of coefficients $\Gamma_{j k}^{i}$. The first-order Riccati PDE system $R_{i j k}^{l}=0$, with the unknowns $\Gamma_{j k}^{i}$, is equivalent to either (i) two first-order PDE relations: the first Riccati PDE relation $\frac{\partial}{\partial x^{p}} \Gamma_{i s}^{l}+\Gamma_{p m}^{l} \Gamma_{i s}^{m} \in k e r(P)$, the second Riccati PDE relation $\frac{\partial}{\partial x^{p}} \Gamma_{i s}^{l}-$ $\Gamma_{s m}^{l} \Gamma_{i p}^{m} \in k e r(P)$; or (ii) two Riccati PDE systems $\frac{\partial}{\partial x^{p}} \Gamma_{i s}^{l}+\Gamma_{p m}^{l} \Gamma_{i s}^{m}=$ symmetric in (ps), $\frac{\partial}{\partial x^{p}} \Gamma_{i s}^{l}-$ $\Gamma_{s m}^{l} \Gamma_{i p}^{m}=$ symmetric in (ps).

Symmetric in ( $p s)$ is equivalent to "the antisymmetric part is zero".

Proof. This result comes from the relations

$$
P_{j k}^{p s}\left(\frac{\partial}{\partial x^{p}} \Gamma_{i s}^{l}+\Gamma_{p m}^{l} \Gamma_{i s}^{m}\right)=P_{j k}^{p s}\left(\frac{\partial}{\partial x^{p}} \Gamma_{i s}^{l}-\Gamma_{s m}^{l} \Gamma_{i p}^{m}\right)=\frac{1}{2} R_{i j k}^{l} .
$$

To the previous tensor curvature-flatness PDE system we attach two non-tensor firstorder strong Riccati PDE systems:

$$
\frac{\partial}{\partial x^{p}} \Gamma_{i s}^{l}(x)+\Gamma_{p m}^{l}(x) \Gamma_{i s}^{m}(x)=0,
$$

and

$$
\frac{\partial}{\partial x^{p}} \Gamma_{i s}^{l}(x)-\Gamma_{s m}^{l}(x) \Gamma_{i p}^{m}(x)=0,
$$

each having as unknown the connection of components $\Gamma_{j k}^{i}$.

Theorem 3. Each of the Riccati PDE systems (1) and (2) is completely integrable.

Proof. The Riccati PDE system (1) is completely integrable since $\partial_{q p}^{2} \Gamma_{i s}^{l}=\partial_{p q}^{2} \Gamma_{i s}^{l}$. For the Riccati PDE system (2) we use the Frobenius Theorem for the Pfaff system

$$
d \Gamma_{i s}^{l}(x)-\Gamma_{s m}^{l}(x) \Gamma_{i p}^{m}(x) d x^{p}=0,
$$

i.e., we prove that there exists an integrant factor. Indeed, the 1 -forms

$$
\omega_{i s}^{l}=\delta_{m}^{l} \delta_{i}^{p} \delta_{s}^{q} d \Gamma_{p q}^{m}(x)-\Gamma_{s m}^{l}(x) \Gamma_{i p}^{m}(x) d x^{p},
$$


on the space $\mathbb{R}^{r}, r=\frac{n^{2}(n+1)}{2}+n$, of coordinates $\left(\Gamma_{p q}^{n}(x), x^{i}\right)$, are smooth and pointwise linearly independent forms (since the matrix of the components has the rank $\frac{n^{2}(n+1)}{2}$ ). Furthermore, there exist the smooth 1-forms $a_{i s}^{l} \alpha \beta$ such that

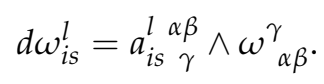

The existence is related to the fact that the number of unknown 1-form components is quite large.

Complete integrability means conditions for the global existence of connection solutions, via Cauchy problems with initial data $\left.\Gamma_{j k}^{i}\left(x^{1}, x^{2}, \ldots, x^{n}\right)\right|_{x^{1}=0}=A_{j k}^{i}\left(x^{2}, \ldots, x^{n}\right)$.

The previous Riccati PDEs (1) and (2) are not tensor equations. That is, they do not have tensor behavior with respect to the changing of the system of coordinates.

If the PDE systems (1) and (2) have a common solution $\Gamma_{j k^{\prime}}^{i}$, then we get a divergence conservation law

$$
\partial_{x^{q}}\left(\delta_{p}^{q} \Gamma_{i s}^{l}+\delta_{s}^{q} \Gamma_{i p}^{l}\right)=0 \text { modulo (1) and (2). }
$$

The exotic object $\delta_{p}^{q} \Gamma_{i s}^{l}+\delta_{s}^{q} \Gamma_{i p}^{l}$ is called the flux of the conservation law.

Remark 2. Given initial conditions $\Gamma_{j k}^{i}\left(0, x^{2}, \ldots, x^{n}\right)=A_{j k}^{i}\left(x^{2}, \ldots, x^{n}\right)$, the Cauchy problem has a unique solution $\Gamma_{j k}^{i}(x)$ in a neighborhood of the point $a=\left(a^{1}, a^{2}, \ldots, a^{n}\right)=(0,0, \ldots, 0)$. An analytic solution can be written like a Taylor series

$$
\Gamma_{j k}^{i}(x)=\Gamma_{j k}^{i}(a)+\partial_{l_{1}} \Gamma_{j k}^{i}(a)\left(x^{l_{1}}-a^{l_{1}}\right)+\frac{1}{2 !} \partial_{l_{1} l_{2}}^{2} \Gamma_{j k}^{i}(a)\left(x^{l_{1}}-a^{l_{1}}\right)\left(x^{l_{2}}-a^{l_{2}}\right)+\ldots
$$

Theorem 4. Any solution $\Gamma_{j k}^{i}(x)$ of one of the Riccati PDE systems (1) and (2) is a solution of the curvature-flatness PDE system.

Proof. This result comes from the relations

$$
P_{j k}^{p s}\left(\frac{\partial}{\partial x^{p}} \Gamma_{i s}^{l}+\Gamma_{p m}^{l} \Gamma_{i s}^{m}\right)=P_{j k}^{p s}\left(\frac{\partial}{\partial x^{p}} \Gamma_{i s}^{l}-\Gamma_{s m}^{l} \Gamma_{i p}^{m}\right)=\frac{1}{2} R_{i j k}^{l} .
$$

Corollary 1. A symmetric connection $\Gamma_{j k}^{i}(x)$ which is a solution of one of the Riccati PDE systems (1) and (2), is the image through a diffeomorphism of the connection $\Gamma_{j k}^{i}(x)=0$.

Corollary 2. Suppose $\Gamma_{i s}^{l}(x)$ is a solution of the PDE system (1) and $B_{i s}^{l}(x)$ is an arbitrary symmetric tensor field. The connection $\Gamma_{i s}^{l}(x)+B_{i s}^{l}(x)$ is a solution of the PDE system (1) if and only if

$$
\frac{\partial}{\partial x^{p}} B_{i s}^{l}(x)+\Gamma_{p m}^{l}(x) B_{i s}^{m}(x)+B_{p m}^{l}(x) \Gamma_{i s}^{m}(x)+B_{p m}^{l}(x) B_{i s}^{m}(x)=0
$$

(Riccati PDEs).

Example 3. For the PDE system (1), there are also nonvanishing solutions such as for example (i) constant connection solutions $\Gamma_{j k}^{i}(x)=C_{j k}^{i}$ if $C_{p m}^{l} C_{i s}^{m}=0$; (ii) connection of the form $\Gamma_{j k}^{i}(x)=C_{j k}^{i} f(x)$; imposing the conditions $C_{i s}^{l}+C_{p m}^{l} C_{i s}^{m}=0$, we find $f(x)=-\frac{1}{x^{1}+\ldots+x^{n}+c}$; given the constants $C_{j k}^{i}$, we obtain $C_{i s}^{l} \frac{d f}{f^{2}}+C_{p m}^{l} C_{i s}^{m} d x^{p}=0$, and $f(x)$ is determined by the formula $C_{i s}^{l}=C_{i s}^{m}\left(C_{1 m}^{l} x^{1}+\ldots+C_{n m}^{l} x^{n}\right) f(x)$; (iii) "self-similar connection" solutions of the form $\Gamma_{j k}^{i}(x)=A_{j k}^{i}(\tilde{\xi}), \xi=\left(x^{1}\right)^{a_{1}} \cdots\left(x^{n}\right)^{a_{n}} ;$ (iv) "soliton connection" solutions (see also [26]) 
$\Gamma_{j k}^{i}(x)=\Phi_{j k}^{i}(u), u=a_{l} x^{l} ;(v)$ solutions of the form $\Gamma_{j k}^{i}=\delta_{j}^{i} f_{k}+\delta_{k}^{i} f_{j}, f(x)=\frac{n+1}{n+3} \ln \left(c_{k} x^{k}+c\right)$ etc. All these connection solutions of the PDE system (1) are solutions for curvature-flatness.

Example 4. Let us consider again the Riccati PDE system (1). In order to obtain a new solution, we use the associated anti-trace PDE system (obtained by applying the anti-summation convention to a pair of dummy indices)

$$
\frac{\partial}{\partial x^{p}} \Gamma_{i s}^{l}(x) \frac{\delta_{k}^{m}}{n}+\Gamma_{p k}^{l}(x) \Gamma_{i s}^{m}(x)=0 .
$$

If we start with the anti-trace Riccati PDE system, by executing the contraction of $m$ with $k$, we obtain again the Riccati PDE system (1). Consequently, any solution of the anti-trace PDE system is solution of the initial PDE system (1).

A solution of the anti-trace system is obtained in the following way. If $m \neq k$, then either $\Gamma_{p k}^{l}(x)=0$ or $\Gamma_{i s}^{m}(x)=0$. If $m=k$, then

$$
\left.\frac{\partial}{\partial x^{p}} \Gamma_{i s}^{l}(x) \frac{1}{n}+\Gamma_{p m}^{l}(x) \Gamma_{i s}^{m}(x)=0 \text { (no sum after } m\right) .
$$

For $n=2$, we obtain a constant connection solution, with certain zero coefficients. Generally, we find that $\Gamma_{i s}^{l}(x)$ must be constant, with certain zero coefficients.

Example 5. Let $n=3$. A curvature-flat constant connection solution of the Riccati PDE system (1), must satisfy

$$
C_{p 1}^{l} C_{i s}^{1}+C_{p 2}^{l} C_{i s}^{2}+C_{p 3}^{l} C_{i s}^{3}=0, l, p, i, s=1,2,3 .
$$

Let us consider the matrix

$$
A_{p}=\left(\begin{array}{ccc}
C_{p 1}^{1} & C_{p 2}^{1} & C_{p 3}^{1} \\
C_{p 1}^{2} & C_{p 2}^{2} & C_{p 3}^{2} \\
C_{p 1}^{3} & C_{p 2}^{3} & C_{p 3}^{3}
\end{array}\right)
$$

If there exists $p$ such that rank $A_{p}=2$, then

$$
\frac{C_{i s}^{1}}{C_{p 2}^{1} C_{p 3}^{2}-C_{p 2}^{2} C_{p 3}^{1}}=\frac{C_{i s}^{2}}{C_{p 1}^{2} C_{p 3}^{1}-C_{p 1}^{1} C_{p 3}^{2}}=\frac{C_{i s}^{3}}{C_{p 1}^{1} C_{p 2}^{2}-C_{p 1}^{2} C_{p 2}^{1}} .
$$

Theorem 5. The following statements are equivalent. (i) The affine manifold $(M, \nabla)$ is curvatureflat, i.e., $R_{i j k}^{l}(x)=0$. (ii) For each point $x \in M$, there exists a chart such that $\Gamma_{j k}^{i}(x)=0$. (iii) For each point $x \in M$, there exists a chart such that $\frac{\partial}{\partial x^{p}} \Gamma_{i s}^{l}(x)=0, \Gamma_{p m}^{l}(x) \Gamma_{i s}^{m}(x)=0$. (iv) For each point $x \in M$, there exists a chart such that $\frac{\partial}{\partial x^{p}} \Gamma_{i s}^{l}(x)+\Gamma_{p m}^{l}(x) \Gamma_{i s}^{m}(x)=0$.

Proof. The diagram

$$
\begin{array}{llr}
(i) & \rightarrow & (i i) \\
\uparrow & & \downarrow \\
(i v) & \leftarrow & (i i i)
\end{array}
$$

is closed. Indeed $(i) \Rightarrow(i i)$ is the classical Theorem 1 . Then the implications ( $i i) \Rightarrow(i i i) \Rightarrow$ (iv) are automatic. The implication $(i v) \Rightarrow(i)$ is Theorem 4.

Remark 3. An equivalent Theorem can be formulated using the Riccati PDE system (2). In this sense Theorem 5 can be replaced by a new Theorem adding the following two statements. (v) For each point in $M$, there exists a chart with local coordinates $\left(x^{1}, \ldots, x^{n}\right)$ such that $\nabla_{\partial_{x^{i}}} \nabla_{\partial_{x j}} \partial_{x^{k}}=0$, for all indices $i, j, k=\overline{1, n}$. (vi) For each point in $M$, there exists a chart with local coordinates $\left(x^{1}, \ldots, x^{n}\right)$ such that $\partial_{x p} \Gamma_{i s}^{l}-\Gamma_{s m}^{l} \Gamma_{i p}^{m}=0$. 
The property (vi) is quite strange, as it is not derived (like (iv)) from a double covariant derivative (like (v)). It deserves a deeper investigation on its own.

Remark 4. If a symmetric connection $\Gamma_{j k}^{i}$ is a solution of curvature-flatness PDEs, i.e., $R^{i}{ }_{j k l}=0$, then the connection $\Gamma_{j^{\prime} k^{\prime}}^{i^{\prime}}$ obtained by an arbitrary changing of coordinates $x^{i^{\prime}}=x^{i^{\prime}}\left(x^{i}\right)$ (diffeomorphism) is a solution of $R_{j^{\prime} k^{\prime} l^{\prime}}=0$, since $R^{i}{ }_{j k l}=0$ is a tensorial equation with the unknown $\Gamma_{j k}^{i}$. According to Theorem 1, for a solution $\Gamma_{j k}^{i}$ of curvature-flatness, there exists a coordinate system where $\Gamma_{j^{\prime} k^{\prime}}^{i^{\prime}}=0$.

Maple offers software that can perform tensor algebra and tensor analysis. If we have a symmetric connection, we can compute the curvature tensor field and then test whether the curvature tensor is zero everywhere. If the curvature tensor is zero everywhere (that is, all its components are zero everywhere), then the manifold is flat. By definition, the manifold is flat if there exists a set of coordinates in which the symmetric connection is everywhere equal to zero.

\subsection{Non-Symmetric Connection Case}

In this subsection, we use the pair $(M, \nabla)$, where $M$ is a smooth real $n$-dimensional $(n \geq 2)$ manifold and $\nabla$ is an affine connection of components $L_{j k}^{i}, i, j, k=\overline{1, n}$, in $\mathcal{F}(M)$, with non-zero torsion $T_{j k}^{i}=L_{j k}^{i}-L_{k j}^{i}$. Although the theory developed here regarding non-symmetric connections is formally similar to that of symmetrical connections, we point out that the nature of the exotic objects used now is totally different.

\subsubsection{Curvature Tensor Field}

The curvature tensor field

$$
L_{i p s}^{l}=\partial_{x^{p}} L_{i s}^{l}-\partial_{x^{s}} L_{i p}^{l}+L_{p m}^{l} L_{i s}^{m}-L_{s m}^{l} L_{i p}^{m}
$$

belongs to the vector space

$$
V=\left\{V_{i p s}^{l}=a \partial_{x p} L_{i s}^{l}+b \partial_{x^{s}} L_{i p}^{l}+c L_{p m}^{l} L_{i s}^{m}+d L_{s m}^{l} L_{i p}^{m} \mid(a, b, c, d) \in \mathbb{R}^{4}\right\} .
$$

The generalized Kronecker delta $\mathcal{P}: V \rightarrow V, \mathcal{P}_{j k}^{p s}=\frac{1}{2}\left(\delta_{j}^{p} \delta_{k}^{s}-\delta_{k}^{p} \delta_{j}^{s}\right)$, is a projector on $V$, i.e., $\mathcal{P}^{2}=\mathcal{P}$, and is covariant constant.

The PDE system $L_{j k l}^{i}=0\left(\frac{n^{2}\left(n^{2}-1\right)}{3}\right.$ PDEs, since the curvature tensor field is antisymmetric in the last two indices and satisfies the first Bianchi identity; and $n^{3}$ unknown functions $L_{j k}^{i}$ ) is equivalent to either a first-order tensor Riccati PDE system written in the form

$$
2 \mathcal{P}_{j k}^{p s} \frac{\partial}{\partial x^{p}} L_{i s}^{l}=L_{k m}^{l} L_{i j}^{m}-L_{j m}^{l} L_{i k}^{m}
$$

or a Riccati PDE system of divergence type

$$
\frac{\partial}{\partial x^{p}}\left(\delta_{j}^{p} L_{i k}^{l}-\delta_{k}^{p} L_{i j}^{l}\right)=L_{k m}^{l} L_{i j}^{m}-L_{j m}^{l} L_{i k}^{m}
$$

In addition, a connection $L_{j k}^{i}(x)$, which satisfies $L_{k m}^{l}(x) L_{i j}^{m}(x)-L_{j m}^{l}(x) L_{i k}^{m}(x)=0$, is curvature-flat solution iff $L_{i k}^{l}(x)=\frac{\partial F_{i}^{l}}{\partial x^{k}}(x)$, with a suitable matrix of elements $F_{i}^{l}(x)$.

Remark 5. For $n=2$, we have a system of 4 PDEs

$$
L_{i 12}^{l}=\partial_{1} L_{i 2}^{l}-\partial_{2} L_{i 1}^{l}+L_{1 m}^{l} L_{i 2}^{m}-L_{2 m}^{l} L_{i 1}^{m}=0, l, i=1,2,
$$

with 8 unknown functions $L_{j k}^{i}, i, j, k=1,2$. This system has an infinity of solutions. 
Remark 6. Let $\Omega_{x_{0} x_{1}} \subset \mathbb{R}^{n}$ be the hyper-parallelepiped fixed by the opposite diagonal points $x_{0}, x_{1} \in \mathbb{R}^{n}$. Let $d v=d x^{1} \wedge \ldots \wedge d x^{n}$ be the volume element in $\mathbb{R}^{n}$.

(i) Open problem. Let us interpret the curvature tensor field $L_{i p s}^{l}$ as a Lagrangian $\mathcal{L}^{l}{ }_{i p s}=\partial_{x^{p}} L_{i s}^{l}-\partial_{x^{s}} L_{i p}^{l}+L_{p m}^{l} L_{i s}^{m}-L_{s m}^{l} L_{i p}^{m}$. Then, we introduce the functionals (total curvature)

$$
I^{l}{ }_{i p s}(L(\cdot))=\int_{\Omega_{x_{0} x_{1}}} \mathcal{L}^{l}{ }_{i p s} d v .
$$

Write explicitly the Euler-Lagrange PDEs

$$
\frac{\partial \mathcal{L}^{l}{ }_{i p s}}{\partial \Gamma_{v w}^{u}}-D_{x^{t}} \frac{\partial \mathcal{L}^{l}{ }_{i p s}}{\partial\left(\partial_{x^{t}} \Gamma_{v w}^{u}\right)}=0 .
$$

Hint:

$$
\frac{\partial L_{j k}^{i}}{\partial L_{m n}^{l}}=\delta_{l}^{i} \delta_{j}^{m} \delta_{k}^{n}, \frac{\partial\left(\partial_{x p} L_{i s}^{q}\right)}{\partial\left(\partial_{x^{r}} L_{m n}^{l}\right)}=\delta_{p}^{r} \delta_{l}^{q} \delta_{i}^{m} \delta_{s}^{n}
$$

(ii) By the Euclidean divergence formula, we evaluate the numbers

$$
\begin{gathered}
I_{k i j}^{l}=\int_{\Omega_{x_{0} x_{1}}}\left(L_{k m}^{l} L_{i j}^{m}-L_{j m}^{l} L_{i k}^{m}\right) d v=\int_{\Omega_{x_{0} x_{1}}} \frac{\partial}{\partial x^{p}}\left(\delta_{j}^{p} L_{i k}^{l}-\delta_{k}^{p} L_{i j}^{l}\right) d v \\
=\int_{\partial \Omega_{x_{0} x_{1}}} \delta_{p q}\left(\delta_{j}^{p} L_{i k}^{l}-\delta_{k}^{p} L_{i j}^{l}\right) n^{q} d \sigma
\end{gathered}
$$

where $n^{q}$ is the unit vector field normal to the boundary $\partial \Omega_{x_{0} x_{1}}$. If $L_{i k}^{l} \mid \partial \Omega_{x_{0} x_{1}}=0$, then $I_{k i j}^{l}=0$.

(iii) Approximate solutions in the sense of the least squares [18]. To find such solutions for the curvature-flatness PDEs $L_{i p s}^{l}=0$ we need a Riemannian metric $g_{i j}$ to create the least squares Lagrangian $\mathcal{L}=\frac{1}{2} g_{l a g} g^{i b} g^{p c} g^{s d} L_{i p s}^{l} L_{b c d}^{a}=\frac{1}{2}\|L\|^{2}$, a compact manifold $M$ to introduce the functional $I\left(L_{v w}^{u}(\cdot)\right)=\int_{M} \mathcal{L} \sqrt{\operatorname{det}\left(g_{i j}\right)} d x^{1} \ldots d x^{n}$, and the Euler-Lagrange PDEs of $\mathcal{L}$ with respect to $L_{v w}^{u}$.

To the curvature-flatness Riccati PDE system $L_{i p s}^{l}=0$, we attach two strong Riccati PDE systems:

$$
\frac{\partial}{\partial x^{p}} L_{i s}^{l}(x)+L_{p m}^{l}(x) L_{i s}^{m}(x)=0,
$$

and

$$
\frac{\partial}{\partial x^{p}} L_{i s}^{l}(x)-L_{s m}^{l}(x) L_{i p}^{m}(x)=0,
$$

whose unknown is the non-symmetric connection $L_{j k}^{i}$. These Riccati PDEs (3) and (4) are not tensor equations and refer to completely different unknowns than PDEs (1) and (2). They are not recoverable with respect to the changing of the system of coordinates.

Theorem 6. Each of the Riccati PDE systems (3) and (4) is completely integrable.

Proof. It is almost identical to that of Theorem 3, although the objects involved are totally different. The space used for reasoning has the dimension $n^{3}+n$.

Complete integrability means conditions for the global existence of connections, via Cauchy problems with initial data

$$
\left.L_{j k}^{i}\left(x^{1}, x^{2}, \ldots, x^{n}\right)\right|_{x^{1}=0}=B_{j k}^{i}\left(x^{2}, \ldots, x^{n}\right) .
$$


Theorem 7. Any solution $L_{j k}^{i}(x)$ of one of the Riccati PDE systems (3) and (4) is a solution of the curvature-flatness PDE system.

Proof. The result is a consequence of the formulas

$$
\mathcal{P}_{j k}^{p s}\left(\frac{\partial}{\partial x^{p}} L_{i s}^{l}+L_{p m}^{l} L_{i s}^{m}\right)=\mathcal{P}_{j k}^{p s}\left(\frac{\partial}{\partial x^{p}} L_{i s}^{l}-L_{s m}^{l} L_{i p}^{m}\right)=\frac{1}{2} L_{i j k}^{l} .
$$

If the PDE systems (3) and (4) have a common solution $L_{j k}^{i}$, then we get a divergence conservation law

$$
\partial_{x^{q}}\left(\delta_{p}^{q} L_{i s}^{l}+\delta_{s}^{q} L_{i p}^{l}\right)=0 \text { modulo (3) and (4). }
$$

The exotic object $\delta_{p}^{q} L_{i s}^{l}+\delta_{s}^{q} L_{i p}^{l}$ is called the flux of the conservation law.

Remark 7. If a non-symmetric connection $L_{j k}^{i}$ is a solution of curvature-flatness PDEs, i.e., $L^{i}{ }_{j k l}=0$, then the connection $L_{j^{\prime} k^{\prime}}^{i^{\prime}}$ obtained by an arbitrary changing of coordinates $x^{i^{\prime}}=x^{i^{\prime}}\left(x^{i}\right)$ (diffeomorphism) is a solution of $L_{j^{\prime} k^{\prime} l^{\prime}}^{i^{\prime}}=0$. On the other hand there exist always connection solutions that do not come from the changing of coordinates.

\subsubsection{Torsion Tensor Field}

The torsion tensor field $T_{j k}^{i}=L_{j k}^{i}-L_{k j}^{i}$ belongs to the vector space

$$
\mathcal{V}=\left\{a L_{j k}^{i}+b L_{k j}^{i} \mid(a, b) \in \mathbb{R}^{2}\right\}
$$

The operator $\mathcal{P}: \mathcal{V} \rightarrow \mathcal{V}, \mathcal{P}_{j k}^{p s}=\frac{1}{2}\left(\delta_{j}^{p} \delta_{k}^{s}-\delta_{k}^{p} \delta_{j}^{s}\right)$ is a projector on $\mathcal{V}$. This projector creates the torsion $T_{j k}^{i}=L_{j k}^{i}-L_{k j}^{i}=2 \mathcal{P}_{j k}^{p s} L_{p s}^{i}$, corresponding to $a=1, b=-1$.

Example 6 (Maple program). > with(DifferentialGeometry);with(Tensor);

$>\operatorname{DGsetup}([x, y, z], M)$

Notation $t:=\& t$.

$M>C 1:=$ Connection $\left(f(y)\left(D_{x} t d x\right) t d y+g(y)\left(D_{x} t d y\right) t d y+h(y)\left(D_{x} t d z\right) t d y\right)$

$$
C 1:=\left(f(y) D_{x} d x\right) d y+\left(\left(g(y) D_{x}\right) d y\right) d y+\left(\left(h(y) D_{x}\right) d z\right) d y
$$

$M>R 1:=$ CurvatureTensor $(\mathrm{C} 1)$;

$$
R 1:=\left(\left(\left(0 D_{x}\right) d x\right) d x\right) d x
$$

Here the manifold is $\mathbb{R}^{3}$ and the curvature-flatness solution is a non-symmetric connection

$$
L_{12}^{1}=f(y), L_{22}^{1}=g(y), L_{32}^{1}=h(y), \text { in rest zero. }
$$

$M>C 2:=$ Connection $\left(f(x)\left(D_{x} t d x\right) t d x+g(y)\left(D_{y} t d y\right) t d y+h(z)\left(D_{z} t d z\right) t d z\right)$

$$
C 2:=\left(f(x) D_{x} d x\right) d x+\left(\left(g(y) D_{y}\right) d y\right) d y+\left(\left(h(z) D_{z}\right) d z\right) d z
$$

$M>R 2:=$ CurvatureTensor $(C 2)$;

$$
R 2:=\left(\left(\left(0 D_{x}\right) d x\right) d x\right) d x
$$

The curvature-flatness solution is

$$
L_{11}^{1}=f(x), L_{22}^{2}=g(y), L_{33}^{3}=h(z), \text { in rest zero, }
$$


on $\mathbb{R}^{3}$.

Example 7. (i) Let us consider an n-dimensional parallelizable manifold $M$. Let $E_{1}, \ldots, E_{n}$ be a basis of vector fields on $M$. One knows there exists a unique affine connection $\nabla^{(-)}$on $M$ such that $\nabla_{E_{i}}^{(-)} E_{j}=0$ for any $i, j=\overline{1, n}$. This Cartan-Schouten connection is flat but, in general, it is not symmetric.

We look for the affine connections satisfying $\nabla_{E_{i}} \nabla_{E_{j}} E_{k}=0$, for all $i, j, k=\overline{1, n}$. Obviously, $\nabla^{(-)}$is such a connection. We derive that $\nabla$ satisfies the previous relations if and only if its components with respect to the given basis verify $E_{i}\left(\Gamma_{j k}^{l}\right)+\Gamma_{j k}^{m} \Gamma_{i m}^{l}=0$, for any $i, j, k, l=\overline{1, n}$. This last relation extends the property (iv) from Theorem 5.

(ii) Let $G$ be an $n$-dimensional Lie group and $E_{1}, \ldots, E_{n}$ be a basis of left invariant vector fields in the Lie algebra $L(G)$. Suppose moreover that the connections are left invariant. Then $\Gamma_{j k}^{m} \Gamma_{i m}^{l}=0$, for any $i, j, k, l=\overline{1, n}$. This shows that the set of all such (left invariant) connections is a "universal" generalized cone in $\mathbb{R}^{n^{3}}$, whose vertex is $\nabla^{(-)}$. In this particular case, the last relation corresponds to the properties (iii) and (iv) from Theorem 5 (it is neither more general nor particular, it is similar).

\section{Curvature-Flat Riemannian Manifolds}

Let us consider an $n$-dimensional Riemannian manifold $\left(M, g=\left(g_{i j}\right)\right)$. The Riemannian metric $\left(g_{i j}\right)$ determines the Levi-Civita connection

$$
\Gamma_{j k}^{i}=\frac{1}{2} g^{i l}\left(\delta_{l}^{r} \delta_{j}^{s} \delta_{k}^{t}+\delta_{l}^{r} \delta_{k}^{s} \delta_{j}^{t}-\delta_{l}^{t} \delta_{j}^{r} \delta_{k}^{s}\right) \frac{\partial g_{r s}}{\partial x^{t}}
$$

and the Riemannian curvature tensor field $\mathrm{Riem}^{g}$ of components

$$
\begin{gathered}
R_{i j k l}=-\frac{1}{2}\left(\frac{\partial^{2} g_{i k}}{\partial x_{j} \partial x^{l}}+\frac{\partial^{2} g_{j l}}{\partial x^{i} \partial x^{k}}-\frac{\partial^{2} g_{j k}}{\partial x^{i} \partial x^{l}}-\frac{\partial^{2} g_{i l}}{\partial x^{j} \partial x^{k}}\right)+g_{a b}\left(\Gamma_{j k}^{a} \Gamma_{i l}^{b}-\Gamma_{j l}^{a} \Gamma_{i k}^{b}\right) \\
=\frac{1}{2} \delta_{[i}^{p} \delta_{j]}^{q} \delta_{[l}^{r} \delta_{k]}^{s} \frac{\partial^{2} g_{p r}}{\partial x^{q} \partial x^{s}}-g_{a b} \delta_{j}^{q} \delta_{i}^{p} \delta_{[l}^{r} \delta_{k]}^{s} \Gamma_{q r}^{a} \Gamma_{p s,}^{b}
\end{gathered}
$$

where

$$
2 P^{r s}{ }_{l k}=\delta_{[l}^{r} \delta_{k]}^{s}=\delta_{l}^{r} \delta_{k}^{s}-\delta_{k}^{r} \delta_{l}^{s} .
$$

In the Riemannian case the connection comes from the metric $g$. Therefore, the previous theory of the zero-curvature connection should go back to the determination of the metric. We prefer to study the zero-curvature equations having as unknown the components of the metric.

In this case Riemannian curvature-flatness is described by the second-order tensor Riccati PDE system

$$
\frac{1}{2} \delta_{[i}^{p} \delta_{j]}^{q} \delta_{[l}^{r} \delta_{k]}^{s} \frac{\partial^{2} g_{p r}}{\partial x^{q} \partial x^{s}}-g_{a b} \delta_{j}^{q} \delta_{i}^{p} \delta_{[l}^{r} \delta_{k]}^{s} \Gamma_{q r}^{a} \Gamma_{p s}^{b}=0,
$$

on $S_{+}^{2} T^{*} M$. This PDE system has $\frac{n^{2}\left(n^{2}-1\right)}{12}$ distinct second-order linear non-homogeneous PDEs whose unknowns are $\frac{n(n+1)}{2}$ functions $g_{i j}$ (positive definite tensor). Details: for $n=2$, undetermined system; for $n>3$, overdetermined system; for $n=3$, determined system. This PDE system is parabolic since the set of eigenvalues of the coefficient tensor $A_{i j l k}^{\text {pqrs }}=\delta_{[i}^{p} \delta_{j]}^{q} \delta_{[l}^{r} \delta_{k]}^{s}$ (tensor product of an operator by itself) contains the eigenvalue 0 . Judging on the vector space $\mathcal{T}_{0}^{4}(M)$, all eigentensors, respectively eigenvalues are: $X^{i j l k}$ symmetric in $(i, j)$ or in $(l, k)$, with $\lambda=0$; $X^{i j l k}$-antisymmetric in $(i, j)$ and in $(l, k)$ with $\lambda=2$. Of course, the previous PDE system has all the properties of a curvature tensor field.

The projection operator $P$ of components $P_{l k}^{r s}=\frac{1}{2}\left(\delta_{l}^{r} \delta_{k}^{s}-\delta_{k}^{r} \delta_{l}^{s}\right)$ has

$$
\operatorname{ker}(P)=\left\{E_{p q k l}, \text { symmetric in }(k l)\right\}, \operatorname{im}(P)=\left\{F_{p q k l} \text {, antisymmetric in }(k l)\right\} .
$$


Consequently, the partial differential relation

$$
\frac{1}{2} \delta_{[i}^{p} \delta_{j]}^{q} \frac{\partial^{2} g_{p r}}{\partial x^{q} \partial x^{s}}-g_{a b} \Gamma_{j r}^{a} \Gamma_{i s}^{b} \in \operatorname{ker}(P),
$$

is equivalent to PDEs

$$
\frac{1}{2} \delta_{[i}^{p} \delta_{j]}^{q} \frac{\partial^{2} g_{p r}}{\partial x^{q} \partial x^{s}}-g_{a b} \Gamma_{j r}^{a} \Gamma_{i s}^{b}=\text { symmetric in (ir) }
$$

The following Cartan-Ambrose-Hicks theorem $[17,27]$ is well known.

Theorem 8. The curvature tensor field $R_{i j k l}$ vanishes if and only if the Riemannian metric $g_{i j}$ is locally Euclidean, i.e., around each point of $M$ there exists a chart such that $g_{i j}=\delta_{i j}$.

Remark 8. Every one-dimensional Riemannian manifold is flat. If $(M, g)$ is a smooth twodimensional connected complete flat Riemannian manifold, then $M$ must be diffeomorphic to $\mathbb{R}^{2}$, $\mathbb{R} \times S^{1}, S^{1} \times S^{1}$, the Möbius strip, or the Klein bottle.

Example 8. The trivial solution of the PDE system $R_{i j k l}=0$ is $g_{i j}(x)=\delta_{i j}$. If a solution $g_{i j}$ is not equal to $\delta_{i j}$, that does not mean the space is not flat. It just means one may be in the wrong coordinates. In other words, if $g_{i j}$ is a solution of the Riccati PDEs system $R_{i j k l}=0$, then the space $(M, g)$ is flat and there exist coordinates such that $g_{i j}=\delta_{i j}$.

In addition, there are "self-similar metric" solutions of the form

$$
g_{i j}(x)=\Phi_{i j}(\xi), \xi=\left(x^{1}\right)^{a_{1}} \cdots\left(x^{n}\right)^{a_{n}},
$$

"soliton metric" solutions $g_{i j}(x)=\Psi_{i j}(u), u=a_{k} x^{k}$, etc., all for curvature-flatness.

Remark 9. The Riccati PDE system $R_{i j k l}=0$ is a tensor system with the unknown $g_{i j}$. If $g_{i j}$ is a solution, then the metric $g_{i^{\prime} j^{\prime}}$ obtained by an arbitrary changing of coordinates $x^{i^{\prime}}=x^{i^{\prime}}\left(x^{i}\right)$ (diffeomorphism) is a solution of $R_{i^{\prime} j^{\prime} k^{\prime} l^{\prime}}=0$.

Theorem 9. For a Riemannian metric $g_{i j}(x)$, the solution of the previous strong Riccati PDEs system is the image through a diffeomorphism of the metric $g_{i j}(x)=\delta_{i j}$.

If we have a Riemannian metric, we can compute the curvature tensor field via Maple and then test whether the curvature tensor is zero everywhere. If the curvature tensor is zero everywhere (that is, all its components are zero everywhere), then the space is flat. By definition, it is flat if there exists a set of coordinates in which the metric tensor is everywhere equal to the Kronecker delta tensor.

Example 9 (Maple program). > with(DifferentialGeometry);with(Tensor):

$>\operatorname{DGsetup}([x, y], M)$

$M>g=A(a x+b y) d x d x+B(a x+b y) d y d y$

$M>C:=$ Christoffel $(g)$

$M>R:=$ CurvatureTensor $(C)$

Soliton metric solution: The manifold is $\mathbb{R}^{2}$ and $R=0$ iff $2 A^{\prime \prime} A B-B^{\prime} A^{\prime} A-A^{\prime 2} B=$ $0, A(u), B(u), u=a x+b y$.

Example: $A(u)=\frac{1}{4}\left(c_{1} u+c_{2}\right)^{2}, B(u)=1$.

$>\operatorname{DGsetup}([x, y, z], M)$

$M>g 1=f(x) d x d x+g(y) d y d y+h(z) d z d z$

$M>C 1:=$ Christoffel $(g 1)$ 


$$
C 1:=\left(\left(\frac{f^{\prime}(x)}{2 f(x)} D_{x}\right) d x\right) d x+\left(\left(\frac{h^{\prime}(y)}{2 h(y)} D_{y}\right) d y\right) d y+\left(\left(\frac{h^{\prime}(z)}{2 h(z)} D_{z}\right) d z\right) d z
$$

$M>R 1:=$ CurvatureTensor $(\mathrm{C} 1)$

$$
R 1=\left(\left(\left(0 D_{x}\right) d x\right) d x\right) d x
$$

Any diagonal metric on $\mathbb{R}^{n}$ is flat.

3.1. Properties of Projection Operator $B=P \otimes P$

We discuss some properties of the operator $B=P \otimes P$ of components $B_{i j l k}^{p q r s}=\frac{1}{4} \delta_{[i}^{p} \delta_{j]}^{q} \delta_{[l}^{r} \delta_{k]}^{s}$.

Proposition 2. We consider the differential 1-form $\omega_{i}(x) d x^{i}$ that determines the four-indexed object $\frac{\partial \omega_{p}}{\partial x^{q}} \frac{\partial \omega_{r}}{\partial x^{s}}$. This object is in $\operatorname{ker}(B)$ if and only if the 1-form $\omega_{i}(x) d x^{i}$ is completely integrable.

Proof. The condition

$$
B_{i j l k}^{p q r s} \frac{\partial \omega_{p}}{\partial x^{q}} \frac{\partial \omega_{r}}{\partial x^{s}}=0
$$

is equivalent to

$$
\left(\frac{\partial \omega_{i}}{\partial x^{j}}-\frac{\partial \omega_{j}}{\partial x^{i}}\right)\left(\frac{\partial \omega_{l}}{\partial x^{k}}-\frac{\partial \omega_{k}}{\partial x^{l}}\right)=0
$$

and hence the differential 1-form $\omega_{i}(x) d x^{i}$ is completely integrable. This means that there exists a function $f$ such that $\omega_{i}(x) d x^{i}=d f(x)$. The hypersurfaces $f\left(x^{1}, \ldots, x^{n}\right)=c$ are solutions of the completely integrable Pfaff equation $\omega_{i}(x) d x^{i}=0$.

Proposition 3. Let us consider the tensor field $\wedge_{i j}$ and the tensor product by itself $\wedge_{p q} \otimes \wedge_{r s}$. The four-indexed tensor $\wedge_{p q} \otimes \wedge_{r s}$ is in $\operatorname{ker}(B)$ if and only if the tensor $\wedge_{i j}$ is symmetric.

Proof. The four-indexed tensor $\wedge_{p q} \otimes \wedge_{r s}$ belongs to $\operatorname{ker}(B)$ if and only if

$$
B_{i j l k}^{p q r s} \wedge_{p q} \wedge_{r s}=0
$$

i.e., $\wedge_{i j}=\wedge_{j i}$ (symmetric).

Proposition 4. The tensor field $\wedge_{i j k l}$ of type $(0,4)$ is in $\operatorname{ker}(B)$ if and only if $\wedge_{i j l k}-\wedge_{i j k l}-\wedge_{j i l k}+$ $\wedge_{j i k l}=0$.

Proof. The tensor field $\wedge_{i j k l}$ of type $(0,4)$ belongs to $\operatorname{ker}(A)$ if and only if

$$
B_{i j l k}^{\text {pqrs }} \wedge \text { pqrs }=0
$$

i.e., $\wedge_{i j l k}-\wedge_{i j k l}-\wedge_{j i l k}+\wedge_{j i k l}=0$.

\subsection{Strong Riccati PDE System}

Any solution $g$ (positive definite tensor), with the components $g_{i j}$, of the strong Riccati PDE system

$$
\frac{1}{2} \delta_{[i}^{p} \delta_{j]}^{q} \frac{\partial^{2} g_{p r}}{\partial x^{q} \partial x^{s}}-g_{a b} \Gamma_{j r}^{a} \Gamma_{i s}^{b}=0,
$$

is a solution of the curvature-flatness PDE system. This second order PDE system is parabolic since the set of eigenvalues of the projection $P_{i j}^{p q}=\frac{1}{2} \delta_{[i}^{p} \delta_{j]}^{q}$ contains 0 . Indeed, 
on $\mathcal{T}_{0}^{2}(M)$, all the eigentensors and eigenvalues are $X^{i j}$-symmetric with $\lambda=0$ and $X^{i j}$ antisymmetric with $\lambda=1$.

Example 10. The two surfaces $(u, v) \rightarrow(u \cos v, u \operatorname{sinv}, \ln u)$ (surface of revolution with exponential curve as meridian-exponential horn) and $(u, v) \rightarrow(u \operatorname{cosv}, u s i n v, v)$ (helicoid) have the same Gauss curvature $-1 /\left(1+u^{2}\right)$ at corresponding points, even though there is no isometric correspondence at corresponding $(u, v)$ points.

Thus even though the two surfaces of revolution (catenoid and exponential horn) share the same Gauss curvature they admit no isometrically mapped correspondence at $(u, v)$.

Example 11 (Maple program). > with(DifferentialGeometry);with(Tensor):

$>\operatorname{DGsetup}([x, y, z], M)$

$M>g=d x d x+\left(1+a(y)^{2}\right) d y d y+2 a(y) d y d z+2 d z d z$

$M>C:=$ Christoffel $(g)$

$$
C:=\frac{a(y) a^{\prime}(y) D_{y}}{1+a(y)^{2}} d y d y-\frac{a^{\prime}(y)(a(y)-1) D_{z}}{2\left(1+a(y)^{2}\right)} d y d y
$$

$M>R:=$ CurvatureTensor $(C)$

$$
R:=0 D_{x} d x d x d x
$$

Result: the Riemannian metric $g$ has zero curvature on $\mathbb{R}^{3}$.

\subsection{Giving the Curvature Tensor Field, Find the Riemannian Metric}

We judge in normal coordinates associated to the Levi-Civita connection.

Giving the curvature tensor $R_{i j k l}$, we look to solve the non-homogeneous second-order Riccati PDEs

$$
\frac{1}{2} \delta_{[i}^{p} \delta_{j]}^{q} \delta_{[l}^{r} \delta_{k]}^{s} \frac{\partial^{2} g_{p r}}{\partial x^{q} \partial x^{s}}-g_{a b} \delta_{j}^{q} \delta_{i}^{p} \delta_{[k}^{r} \delta_{l]}^{s} \Gamma_{q r}^{a} \Gamma_{p s}^{b}=R_{i j k l},
$$

with respect to $g_{i j}$, trying for series solution

$$
g_{j l}(x)=g_{j l}(0)+\frac{\partial g_{j l}}{\partial x^{k}}(0) x^{k}+\frac{1}{2} \frac{\partial^{2} g_{j l}}{\partial x^{i} \partial x^{k}}(0) x^{i} x^{k}+\ldots
$$

At the center of normal coordinates, the components of curvature tensor field Riem ${ }^{g}$ are

$$
R_{i j k l}(0)=-\frac{1}{2}\left(\frac{\partial^{2} g_{i k}}{\partial x^{j} \partial x^{l}}+\frac{\partial^{2} g_{j l}}{\partial x^{i} \partial x^{k}}-\frac{\partial^{2} g_{j k}}{\partial x^{i} \partial x^{l}}-\frac{\partial^{2} g_{i l}}{\partial x^{j} \partial x^{k}}\right)(0) .
$$

Now we observe that the Cauchy problem (strong normal second-order PDE system with initial data)

$$
\partial_{i k}^{2} g_{j l}=\frac{1}{3}\left(R_{i j k l}+R_{i l k j}\right), \quad g_{i j}(0)=\delta_{i j}, \quad \partial_{k} g_{i j}(0)=0
$$

determines a solution $g_{j l}(x)$ for our initial problem.

Indeed, given $R_{i j k l}$, any smooth metric of the form

$$
g_{j l}(x)=g_{j l}(0)+\frac{1}{3}\left(R_{i j k l}+R_{i l k j}\right) x^{i} x^{k}+O\left(|x|^{3}\right)
$$

has curvature tensor $R_{i j k l}$ at $x=0$.

\section{Invariant Version for Curvature-Flatness}

A permutation $\sigma$ of the set $\{1,2,3\}$ determines a mapping $\sigma: \mathcal{X}(M)^{3} \rightarrow \mathcal{X}(M)^{3}$, $\sigma\left(X_{1}, X_{2}, X_{3}\right)=\left(X_{\sigma(1)}, X_{\sigma(2)}, X_{\sigma(3)}\right)$ of the 3-th Cartesian power of $\mathcal{X}(M)$. Let $\varphi: \mathcal{X}(M)^{3} \rightarrow$ 
$\mathcal{X}(M)^{\otimes 3}$ be the natural multilinear embedding of the Cartesian product of $\mathcal{X}(M)$ into the tensor power of $\mathcal{X}(M)$. Then, by the universal property, there is a unique isomorphism $\tau_{\sigma}: \mathcal{X}(M)^{\otimes 3} \rightarrow \mathcal{X}(M)^{\otimes 3}$ such that $\varphi \circ \sigma=\tau_{\sigma} \circ \varphi$. The isomorphism $\tau_{\sigma}$ is called the braiding map associated to the permutation $\sigma$ and $\mathcal{X}(M)^{3} \simeq \mathcal{X}(M)^{\otimes 3}$.

Let us introduce a product structure diagram

$$
\begin{array}{ccc}
(X, Y, Z) \stackrel{p}{\longrightarrow} & (Y, X, Z) \\
\searrow \text { id } & \downarrow p \\
& (X, Y, Z)
\end{array}
$$

It follows that $p^{2}=i d$. The curvature tensor field $R^{\nabla}$ and the product structure $p$ determine the diagram

$$
(X, Y, Z) \quad \stackrel{R^{\nabla}}{\longrightarrow} \quad \begin{array}{cc}
R^{\nabla}(X, Y) Z \\
\searrow-R^{\nabla} & \downarrow p \\
& -R^{\nabla}(X, Y) Z
\end{array}
$$

Consequently, $p \circ R^{\nabla}=-R^{\nabla}$.

For any exotic object $E$, we call $p \circ E$ as $E$ permuted by $p$. The product structure $p$ determines a projector $Q$. To show that, we use an exotic geometrical object $E(X, Y, Z)$. By definition

$$
p \circ E(X, Y, Z) \stackrel{\text { def }}{=} E(p(X, Y, Z))=E(Y, X, Z) .
$$

Since $p$ is a product structure, the object $Q=\frac{1}{2}(i d-p)$ is a projector. Moreover $(i d-p) \circ E(X, Y, Z)=i d \circ E(X, Y, Z)-p \circ E(X, Y, Z)=E(X, Y, Z)-E(p(X, Y, Z))=$ $E(X, Y, Z)-E(Y, X, Z)$.

Although in the invariant version the PDEs are hidden by the way of expression (sense of formulas), this version still offers new points of view on the problems of curvatureflatness. This point of view is not very accessible for those who are specialists in PDEs.

\subsection{Symmetric Connection Case}

In this subsection, we use the pair $(M, \nabla)$, where $M$ is a smooth real $n$-dimensional $(n \geq 2)$ manifold and $\nabla$ is a symmetric affine connection.

The torsion tensor field is $T^{\nabla}(X, Y)=\nabla_{X} Y-\nabla_{Y} X-[X, Y]=0$. We use the vector space

$$
V=\left\{V(X, Y, Z)=a \nabla_{X} \nabla_{Y} Z+b \nabla_{Y} \nabla_{X} Z+c \nabla_{\nabla_{Y} X} Z+d \nabla_{\nabla_{X} Y} Z \mid(a, b, c, d) \in \mathbb{R}^{4}\right\}
$$

of all linear combinations of given vector functions (linear covering). An element (exotic object) $V(X, Y, Z)$ is identified to $(a, b, c, d) \in \mathbb{R}^{4}$. The curvature tensor field

$$
R^{\nabla}(X, Y) Z=\nabla_{X} \nabla_{Y} Z-\nabla_{Y} \nabla_{X} Z+\nabla_{\nabla_{Y} X} Z-\nabla_{\nabla_{X} Y} Z
$$

belongs to the set $V$, corresponding to $(1,-1,1,-1) \in \mathbb{R}^{4}$. The set of all elements having symmetries like the curvature tensor field is a vector subspace $W$. In addition, $Q \circ R^{\nabla}=R^{\nabla}$ (eigentensor $=R^{\nabla}$, eigenvalue $=1$ ).

Theorem 10. The following statements are equivalent.

(i) The diagram

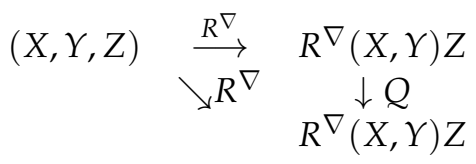

is commutative.

(ii) The curvature tensor field $R^{\nabla}$ is an eigentensor of projector field $Q$, with the eigenvalue 1 . 
Proof. The curvature tensor field $R^{\nabla}$ and the projector $Q$ determine the commutative diagram in the proposition. Since $Q \circ R^{\nabla}=R \nabla$, the curvature tensor field $R \nabla$ is an eigentensor of the projector $Q$, with eigenvalue 1 .

Theorem 11. Let $\nabla$ be a symmetric connection of components $\Gamma_{j k}^{i}$ as an unknown. Denote $A(X, Y, Z)=\nabla_{X} \nabla_{Y} Z+\nabla_{\nabla_{Y} X} Z$ and $B(X, Y, Z)=\nabla_{X} \nabla_{Y} Z-\nabla_{\nabla_{X} Y} Z$. The following firstorder PDEs in the unknown $\Gamma_{j k}^{i}$ are equivalent to each other, and equivalent to partial differential relations:

(i) The PDE $R^{\nabla}(X, Y) Z=0, \forall X, Y, Z \in \mathcal{X}(M)$; (ii) The Riccati PDEs $Q \circ A(X, Y, Z)=0$, $\forall X, Y, Z \in \mathcal{X}(M)$ or $Q \circ B(X, Y, Z)=0, \forall X, Y, Z \in \mathcal{X}(M)$; (iii) The Riccati partial differential relations $\nabla_{X} \nabla_{Y} Z+\nabla_{\nabla_{Y} X} Z \in \operatorname{ker}(Q), \forall X, Y, Z \in \mathcal{X}(M)$ or $\nabla_{X} \nabla_{Y} Z-\nabla_{\nabla_{X} Y} Z \in$ $\operatorname{ker}(Q), \forall X, Y, Z \in \mathcal{X}(M)$; (iv) The Riccati PDEs $\nabla_{X} \nabla_{Y} Z+\nabla_{\nabla_{Y} X} Z=$ symmetric in $(X, Y)$, $\forall X, Y, Z \in \mathcal{X}(M)$ or $\nabla_{X} \nabla_{Y} Z-\nabla_{\nabla_{X}} Z=$ symmetric in $(X, Y), \forall X, Y, Z \in \mathcal{X}(M)$.

Proof. The exotic terms

$$
A(X, Y, Z)=\nabla_{X} \nabla_{Y} Z+\nabla_{\nabla_{Y} X} Z, \quad B(X, Y) Z=\nabla_{X} \nabla_{Y} Z-\nabla_{\nabla_{X} Y} Z,
$$

from the curvature tensor field, are not $\mathcal{F}(M)$-linear, i.e., are not tensor fields. For $Q=\frac{1}{2}(i-p)$, it follows that

$$
Q \circ A(X, Y, Z)=\frac{1}{2} R^{\nabla}(X, Y) Z, Q \circ B(X, Y, Z)=\frac{1}{2} R^{\nabla}(X, Y) Z .
$$

Remark 10. The Riccati PDEs in Theorems 2 and 11 are not the same. Indeed, the components of the curvature tensor field are

$$
\begin{gathered}
\nabla_{\partial_{i}} \nabla_{\partial_{j}} \partial_{k}-\nabla_{\partial_{j}} \nabla_{\partial_{i}} \partial_{k}+\nabla_{\nabla_{\partial_{i}} \partial_{j}} \partial_{k}-\nabla_{\nabla_{\partial_{j}} \partial_{i}} \partial_{k} \\
=\nabla_{\partial_{i}}\left(\Gamma_{j k}^{a} \partial_{a}\right)-\nabla_{\partial_{j}}\left(\Gamma_{i k}^{a} \partial_{a}\right)+\nabla_{\Gamma_{i j}^{a} \partial_{a}} \partial_{k}-\nabla_{\Gamma_{j i}^{a} \partial_{a}} \partial_{k} \\
=\left(\partial_{i} \Gamma_{j k}^{a}+\Gamma_{j k}^{m} \Gamma_{i m}^{a}+\Gamma_{i j}^{m} \Gamma_{m k}^{a}\right) \partial_{a}-\left(\partial_{j} \Gamma_{i k}^{a}+\Gamma_{i k}^{m} \Gamma_{j m}^{a}+\Gamma_{j i}^{m} \Gamma_{m k}^{a}\right) \partial_{a} .
\end{gathered}
$$

The end terms in parentheses are reduced by the symmetry of the connection.

\subsection{Non-Symmetric Connection Case}

In this subsection, we use the pair $(M, \nabla)$, where $M$ is a smooth real $n$-dimensional $(n \geq 2)$ manifold and $\nabla$ is an affine connection with non-zero torsion.

\subsubsection{Curvature Tensor Field}

The curvature tensor field $R^{\nabla}(X, Y) Z=\nabla_{X} \nabla_{Y} Z-\nabla_{Y} \nabla_{X} Z-\nabla_{[X, Y]} Z$ can be rewritten

$$
R^{\nabla}(X, Y) Z=\nabla_{X} \nabla_{Y} Z-\nabla_{Y} \nabla_{X} Z+\nabla_{\nabla_{Y} X} Z-\nabla_{\nabla_{X} Y} Z+\nabla_{T(X, Y)} Z \text {. }
$$

It belongs to the vector space

$$
V=\left\{V(X, Y, Z)=a \nabla_{X} \nabla_{Y} Z+b \nabla_{Y} \nabla_{X} Z+c \nabla_{\nabla_{Y} X} Z+d \nabla_{\nabla_{X} Y} Z+e \nabla_{T(X, Y)} Z\right\},
$$

of all linear combinations of given vector functions (linear covering). Here an element (exotic object) $V(X, Y, Z)$ is identified to $(a, b, c, d, e) \in \mathbb{R}^{5}$. The curvature tensor field corresponds to $(1,-1,1,-1,1) \in \mathbb{R}^{5}$. The set of all elements having symmetries like the curvature tensor field is a vector subspace $W$ of the vector space $V$. 
Remark 11. The vector space $V$ was created to give a sense to the projector $Q: V \rightarrow V$. Of course, there are also other vector spaces giving sense to similar projectors. For example, starting with $Q$ and taking into consideration $A_{3}(X, Y, Z)=[[X, Y], Z]$, we find in addition the property $Q \circ A_{3}(X, Y, Z)=A_{3}(X, Y, Z)$. Of course, $[[X, Y], Z]$ is not linear on $\mathcal{F}(M)$.

Theorem 12. The following statements are equivalent.

(i) The diagram

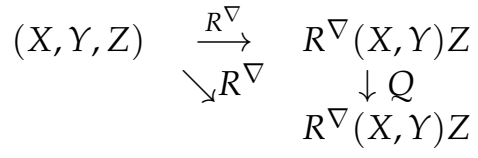

is commutative.

(ii) The curvature tensor field $R \nabla$ is an eigentensor of projector field $Q$, with the eigenvalue 1 .

Theorem 13. Let $\nabla$ be a connection, with torsion $T$, of components $L_{j k}^{i}$ as unknowns. Denote $A_{1}(X, Y, Z)=\nabla_{X} \nabla_{Y} Z+\nabla_{\nabla_{Y} X} Z+\frac{1}{2} \nabla_{T(X, Y)} Z$ and $B_{1}(X, Y, Z)=\nabla_{X} \nabla_{Y} Z-\nabla_{\nabla_{X}} Z+$ $\frac{1}{2} \nabla_{T(X, Y)} Z$. The following first-order PDEs are equivalent to each other and equivalent to firstorder partial differential inclusions:

(i) The PDE $R^{\nabla}(X, Y) Z=0, \forall X, Y, Z \in \mathcal{X}(M)$; (ii) The Riccati PDEs $Q \circ A_{1}(X, Y, Z)=0$, $\forall X, Y, Z \in \mathcal{X}(M)$ or $Q \circ B_{1}(X, Y, Z)=0, \forall X, Y, Z \in \mathcal{X}(M)$; (iii) The Riccati partial differential relations $\nabla_{X} \nabla_{Y} Z+\nabla_{\nabla_{Y} X} Z+\frac{1}{2} \nabla_{T(X, Y)} Z \in \operatorname{ker}(Q), \forall X, Y, Z \in \mathcal{X}(M)$ or $\nabla_{X} \nabla_{Y} Z-$ $\nabla_{\nabla_{X} Y} Z+\frac{1}{2} \nabla_{T(X, Y)} Z \in \operatorname{ker}(Q), \forall X, Y, Z \in \mathcal{X}(M)$; (iv) The Riccati PDEs $\nabla_{X} \nabla_{Y} Z+\nabla_{\nabla_{Y} X} Z+$ $\frac{1}{2} \nabla_{T(X, Y)} Z=$ symmetric in $(X, Y), \forall X, Y, Z \in \mathcal{X}(M)$ or $\nabla_{X} \nabla_{Y} Z-\nabla_{\nabla_{X} Y} Z+\frac{1}{2} \nabla_{T(X, Y)} Z=$ symmetric in $(X, Y), \forall X, Y, Z \in \mathcal{X}(M)$.

Proof. The exotic terms $A_{1}(X, Y, Z)=\nabla_{X} \nabla_{Y} Z+\nabla_{\nabla_{Y} X} Z+\frac{1}{2} \nabla_{T(X, Y)} Z, B_{1}(X, Y, Z)=$ $\nabla_{X} \nabla_{Y} Z-\nabla_{\nabla_{X} Y} Z+\frac{1}{2} \nabla_{T(X, Y)} Z$ from the curvature tensor field are not $\mathcal{F}(M)$-linear, i.e., are not tensor fields. It follows that $Q \circ A(X, Y, Z)=\frac{1}{2} R^{\nabla}(X, Y) Z, Q \circ B(X, Y, Z)=$ $\frac{1}{2} R^{\nabla}(X, Y) Z$.

\subsubsection{Torsion Tensor Field}

The torsion tensor field is defined by $T^{\nabla}(X, Y)=\nabla_{X} Y-\nabla_{Y} X-[X, Y]$. It belongs to the vector space $\mathcal{V}=\left\{a \nabla_{X} Y+b \nabla_{Y} X+c[X, Y] \mid(a, b, c) \in \mathbb{R}^{3}\right\}$. By definition $\mathcal{F} \circ$ $E(X, Y) \stackrel{\text { def }}{=} E(\mathcal{F}(X, Y))=E(Y, X)$. The projector $\mathcal{P}=\frac{1}{2}(I-\mathcal{F}): \mathcal{V} \rightarrow \mathcal{V}$ satisfies $\mathcal{P}\left(\nabla_{X} Y-\frac{1}{2}[X, Y]\right)=\mathcal{P}\left(-\nabla_{Y} X-\frac{1}{2}[X, Y]\right)=\frac{1}{2} T(X, Y)$.

\section{Conclusions}

The curvature-flatness problem is an important geometric problem in Riemannian geometry and differential geometry based on Riemannian metrics or on affine connections. In this paper we studied strong Riccati PDEs and partial differential relations, which imply the flatness Riccati PDEs. The index form technique facilitates the understanding of the significance of the geometric PDEs correlated with curvature-flatness.

We put all components of the PDEs together into "tensor notation" and so forth. Now if anyone is interested in performing the computations in differential geometry and related PDEs, there are Maple packages.

For all the above geometric PDEs, only the existence and uniqueness of solutions have been discussed. Particularly, we have referred to tensor and non-tensor PDEs of the Riccati type, whose unknowns are the components of an affine connection or a Riemannian metric. The Riccati PDEs play a very important role in differential geometry and dynamical systems. 
Our paper treats geometric approaches to specific partial differential equations. Geometry in this context means that certain results do not depend on the coordinate choices made to write down a PDE, and also that structures like connections or metrics are unknowns in PDEs. The examples are significant.

Our aim was to contribute to the debate on curvature-flatness, proposing original ideas carried out from different approaches and perspectives, and linked to other relevant topics within differential geometry and PDEs. As is usual in differential geometry, our paper is calculation intensive: symbols, indices, awe-inspiring PDEs, suggested solutions, etc.

Author Contributions: Conceptualization, I.H., C.U., G.P. and I.T.; methodology, all authors; writingoriginal draft preparation, I.H., C.U., G.P. and I.T.; writing-review and editing, I.H., C.U., G.P. and I.T.; validation, C.U. All authors have read and agreed to the published version of the manuscript.

Funding: This research received no external funding.

Institutional Review Board Statement: Not applicable.

Informed Consent Statement: Not applicable.

Data Availability Statement: Not applicable.

Acknowledgments: We are grateful to reviewers for interesting proposals on improving our point of view.

Conflicts of Interest: The authors declare no conflict of interest.

\section{References}

1. Evans, L.C. Partial Differential Equations; American Mathematical Society: Providence, RI, USA, 1998.

2. Eastwood, M. Notes on Projective Differential Geometry. Symmetries and Overdetermined Systems of Partial Differential Equations (Minneapolis, MN, 2006); Springer: New York, NY, USA, 2007; pp. 41-61.

3. Eastwood, M.; Matveev, V.S. Metric Connections in Projective Differential Geometry. Symmetries and Overdetermined Systems of Partial Differential Equations, (Minneapolis, MN, 2006); Springer: New York, NY, USA, 2007; pp. 339-351.

4. Gurski, M.J.; Viaclovsky, J.A. Fully nonlinear equations on Riemannian manifolds with negative curvature. Indiana U. Math. J. 2003, 52, 399-419. [CrossRef]

5. Hirica, I.; Udriste, C. Basic evolution PDEs in Riemannian geometry. Balkan J. Geom. Appl. 2012, 17, 30-40.

6. Kobayashi, S.; Nomizu, K. Foundations of Differential Geometry; I, Interscience Publ.: Geneva, Switzerland,1963.

7. Nomizu, K.; Sasaki, T. Affine Differential Geometry; Cambridge Univ. Press: Cambridge, UK, 1994.

8. Spencer, D. Overdetermined systems of linear partial differential equations. Bull. Am. Math. Soc. 1969, 75, 179-239. [CrossRef]

9. Udriste, C.; Tevy, I. Geometric dynamics on Riemannian manifolds. Mathematics 2020, 8, 79. [CrossRef]

10. Yau, S.-T. The role of Partial Differential Equations in Differential Geometry. In Proceedings of the International Congress of Mathematicians, Helsinki, Finland, 15-23 August 1978.

11. Blasi, F.S.D.; Pianigiani, G. On the Dirichlet problem for first order partial differential equations. A Baire category approach. NoDEA Nonlinear Diff. Eq. Appl. 1999, 6, 13-34. [CrossRef]

12. Pianigiani, G. Differential inclusions. The Baire category method. In Methods of Nonconvex Analysis; Cellina, A., Ed.; Lecture Notes in Math. 1446; Springer: Berlin, Germany, 1990; pp. 104-136.

13. Charlap, L.S. Compact flat Riemannian manifolds, I. Ann. Math. 1965, 81, 15-30. [CrossRef]

14. Charlap, L.S.; Vasquez, A.T. Compact flat Riemannian manifolds, II. Amer. J. Math. 1965, 87, 551-563. [CrossRef]

15. Charlap, L.S.; Vasquez, A.T. Compact flat Riemannian manifolds III: The group of affinities. Amer. J. Math. 1973, 95, 471-494. [CrossRef]

16. DeTurck, D.; Kazdan, J.L.; Jerry, L. Some regularity theorems in Riemannian geometry. Ann. Sci. Éc. Norm. Supér. 1981, 14, 249-260. [CrossRef]

17. DeTurk, D.; Yang, D. Local existence of smooth metrics with prescribed curvature. Contemp. Math. 1986, 51, 37-43.

18. Hirica, I.; Udriste, C.; Pripoae, G.; Tevy, I. Least squares approximation of flatness on Riemannian manifolds. Mathematics 2020, 8 , 1757. [CrossRef]

19. Vasquez, A.T. Flat Riemannian manifolds. J. Diff. Geom. 1970, 4, 367-382. [CrossRef]

20. Bracken, P. Connections defining representations of zero curvature and their Lax and Bäcklund mappings. J. Geom. Phys. 2013, 70, 157-163. [CrossRef]

21. Bryant, R.L.; Manno, G.; Matveev, V.S. A solution of a problem of Sophus Lie: Normal forms of two-dimensional metrics admitting two projective vector fields. Math. Ann. 2008, 340, 437-463. [CrossRef]

22. Donaldson, N.; Terng, C. Conformally flat submanifolds in spheres and integrable systems. Tohoku Math. J. 2011, 63, 277-302 [CrossRef]

23. Krupka, D. Natural Projectors in Tensor Spaces. Contrib. Algebra Geom. 2002, 43, 217-231. 
24. Qi, L.; Chen, H.; Chen, Y. Tensor Eigenvalues and Their Applications (Advances in Mechanics and Mathematics, 39); Springer: Berlin, Germany, 2018.

25. Bejenaru, A.; Udriste, C. Multivariate optimal control in Riemannian setting. Symmetry Spec. Issue Geom. Submanifolds Homog. Spaces 2019, 11, 893.

26. Rogers, C.; Schief, W.K. Backlund and Darboux Transformations, Geometry and Modern Applications in Soliton Theory; Cambridge University Press: Cambridge, UK, 2002.

27. Lee, J.M. Riemannian Manifolds, An Introduction to Curvature; Springer: Berlin, Germany, 1997. 